\title{
Kommission Schwangerschaftsultraschall
}

\author{
Änderung: \\ Adresse des Kommissions-Sekretariates
}

Für sämtliche Belange des Fähigkeitsausweises Schwangerschaftsultraschall und die Zertifizierung sowie Audit in Ersttrimesterultraschall ist das Sekretariat der Kommission Schwangerschaftsultraschall zuständig.

Ab 1. Januar 2013 ist das Kommissionssekretariat im SGUM/SSUM-Sekretariat in Zürich integriert, Kommissions-Präsidentin ist Frau Prof. Dr. Sevgi Tercanli in Basel. Ab diesem Datum sind neue Anträge an diese Adresse zu richten.

Die laufenden Anfragen und Anträge, die an das bisherige Kommissionssekretariat in Chur gesandt wurden, werden auch noch dort bearbeitet und brauchen nicht erneut an die neue Sekretariatsadresse eingereicht werden.

Für die Kommission Schwangerschaftsultraschall

PD Dr. K. Biedermann

Kantonsspital Graubünden

7000 Chur

$\begin{array}{ll}\text { Wechsel Sekretariat der Kommission } \\ \text { Schwangerschaftsultraschall } \\ \text { Bisher: } & \text { Kommissionssekretariat } \\ & \text { PD Dr. K. Biedermann } \\ & \text { Kantonsspital GR } \\ & 7000 \text { Chur } \\ \text { Neu: } & \text { Info und Erstkontakt: } \\ & \text { Sekretariat SGUM/SSUM } \\ & \text { Postfach 1816, } 8021 \text { Zürich } \\ & \text { Telelefon: 044 } 8251133 \\ & \text { E-Mail: SGUM@hin.ch } \\ & \text { Unterlagen einreichen: } \\ & \text { Frau Prof. Dr. S. Tercanli } \\ & \text { Ultraschall-Praxis Freie Strasse } 38 \\ & \text { 4001 Basel } \\ & \text { E-Mail: sevgi.tercanli@ } \\ \text { highspeed.ch } \\ \text { Telefon: } 0612602880\end{array}$

Wechsel Sekretariat der Kommission

Schwangerschaftsultraschal

PD Dr. K. Biedermann

Kantonsspital GR

Info und Erstkontakt:

Sekretariat SGUM / SSUM

Postfach 1816, 8021 Zürich

Frau Prof. Dr. S. Tercanli

Ultraschall-Praxis Freie Strasse 38

E-Mail: sevgi.tercanli@

highspeed.ch 